\title{
Screening of disorder due to interaction near the Mott transition
}

Quantum Ripples in Strongly Correlated Metals

Phys. Rev. Lett. 104, 236401 (2010), by E. C. Andrade, E. Miranda, and V. Dobrosavljevic

Energy-Resolved Spatial Inhomogeneity of Disordered Mott Systems

Physica B 404, 3167 (2009), by E. C. Andrade, E. Miranda, and V. Dobrosavljevic

Electronic Griffiths Phase of the $d=2$ Mott Transition

Phys. Rev. Lett. 102, 206403 (2009), by E. C. Andrade, E. Miranda, and V. Dobrosavljevic

\section{Recommended and a Commentary by Matthias Vojta, TU Dresden, Germany}

Understanding the combined effect of disorder and electron-electron interactions constitutes one of the most challenging problems in theoretical condensed matter physics. It is also of immense experimental relevance, because (i) many correlated-electron materials are intrinsically disordered due to chemical doping, with transition-metal oxides like cuprate high-temperature superconductors being prime examples, and (ii) a variety of non-trivial effects in two-dimensional (2d) electron systems, such as fractional quantum Hall states and many metal-insulator transitions, can be attributed to an interplay of disorder and interactions.

Perturbative studies of the disordered interacting electron gas go back to the seminal work of Altshuler and Aronov [1], and recent extensions include the two-loop large- $N$ renormalizationgroup (RG) analysis of Punnoose and Finkelstein [2] which allowed to describe a metal-insulator transition in $2 \mathrm{~d}$. However, it is clear that a number of relevant phenomena are beyond the reach of perturbative methods - this includes the interaction-driven metal-insulator (i.e. Mott) transition and its fate upon carrier doping, by many believed to be central to the physics of cuprates. In general, non-perturbative results for disordered interacting systems are difficult to obtain and therefore scarce.

In a series of papers, Andrade and co-workers have theoretically investigated the fate of quenched disorder in two-dimensional lattice systems with strong electron-electron interactions. The Hamiltonian is a standard Hubbard model, but with random on-site energies $\epsilon_{i}$ :

$$
\mathcal{H}=\sum_{i \sigma} \epsilon_{i} c_{i \sigma}^{\dagger} c_{i \sigma}-t \sum_{\langle i j\rangle \sigma}\left(c_{i \sigma}^{\dagger} c_{j \sigma}+\text { h.c. }\right)+U \sum_{i} n_{i \uparrow} n_{i \downarrow} .
$$

Their approach is based on a generalization of dynamical mean-field theory (DMFT) [3] to spatially inhomogeneous systems, dubbed statistical DMFT (or real-space DMFT) [4]. Here, the electronic self-energy is approximated as a local, but site-dependent, quantity $\Sigma_{i j}(\omega) \approx \Sigma_{i i}(\omega) \delta_{i j}$, where $i, j$ denote lattice sites. This approximation scheme reduces the lattice model (1) to a collection of coupled single-impurity models to be solved self-consistently for every realization of disorder - still a numerically challenging task. While numerically exact solutions of real-space 
DMFT equations have been obtained for disorder-free problems [5], Andrade and co-workers have employed a further (slave-boson) approximation to solve the quantum impurity models, known to work well in the low-temperature limit. Importantly, their methodology captures interaction-induced local physics (i.e. formation and screening of local moments) and disorderinduced spatial variations in the single-particle sector and thus is well suited to deal with both Mott and Anderson localization.

The most important results of Andrade and co-workers, obtained by placing a half-filled system on the metallic side of the Mott transition, can be summarized as follows:

(i) The effective on-site disorder, as measured by the variation of renormalized site energies, is very efficiently screened by interactions. This can be rationalized [6] in terms of Kondo physics which governs the strongly correlated Fermi liquid [3]: The Kondo resonance remains pinned to the Fermi level even under sizeable variations of the bare on-site energy.

(ii) In contrast, strong spatial variations are generated in quantities measuring the local coherence, such as the local quasiparticle weight or the local spin susceptibility (both of which can be easily extracted from real-space DMFT). Qualitatively, this can as well be understood in the Kondo picture where the local Kondo scale depends sensitively on local quantities (like the on-site energy). Remarkably, these spatial variations diverge upon approaching the Mott transition, in the sense that the distributions develop a power-law tail at small weights (or large susceptibilities). This tail originates from rare regions which form local "Mott droplets" inside the metallic phase and dominate the thermodynamic response at low energies - a behavior very similar to quantum Griffiths singularities [7].

(iii) The interplay of (i) and (ii) has dramatic consequences, e.g., for the local density of states: It tends to be homogeneous near the Fermi level, but develops strong inhomegeneities away from it (i.e. on a scale set by the overall coherence scale). Phenomenologically, this is very similar to observations in STM studies of cuprate superconductors [8].

One may ask about the relation between these results and the existing perturbative work, e.g., of Punnoose and Finkelstein [2], the common aspect being that effects of disorder are reduced through interactions. On the one hand, it seems clear that most of the physics described by Andrade et al. is not contained in the perturbative approach: The latter cannot capture the formation and subsequent Kondo screening of local moments which underlies the real-space DMFT results. Moreover, the perturbative calculation is not sensitive to rare events which dominate the Griffiths-like regime near the Mott transition. On the other hand, the work of Andrade et al. has, at present, little to say about the fate of Anderson localization in the presence of interactions, because the numerical calculations have to be performed on rather small finite-size systems, such that localization on large length scales is not detectable. Finally, a conceptual difference is that the theory of Andrade et al. is for a half-filled lattice, while the RG theory of Ref. [2] is for a continuum system. (The latter limit appears appropriate for $2 \mathrm{~d}$ MOSFETs due to the low carrier concentration in the experiments.)

It is interesting to ask how much of the strong-coupling described by Andrade et al. survives off half filling. While, far away from half filling, there is little room for Mott-like physics in the 
weak-disorder case, this may change for strong disorder, where interactions can convert localized electrons into local moments. In fact, local-moment formation is believed to be important near the disorder-driven metal-insulator transition, e.g., in the $3 \mathrm{~d}$ doped semiconductor Si:P [9, 10], but a detailed understanding of how the moments affect the transition is lacking. Extending the work of Andrade et al. to arbitrary band filling will therefore be an important step.

\section{References}

[1] B. L. Altshuler and A. G. Aronov, Modern Problems in Condensed Matter Physics (Elsevier, 1985), Chap. X, p. 1.

[2] A. Punnoose and A. M. Finkelstein, Science 310, 289 (2005).

[3] A. Georges, G. Kotliar, W. Krauth, and M. J. Rozenberg, Rev. Mod. Phys. 68, 13 (1996).

[4] V. Dobrosavljevic and G. Kotliar, Phys. Rev. Lett. 78, 3943 (1997).

[5] R. W. Helmes, T. A. Costi, and A. Rosch, Phys. Rev. Lett. 100, 056403 (2008).

[6] D. Tanaskovic, V. Dobrosavljevic, E. Abrahams, and G. Kotliar, Phys. Rev. Lett. 91, 066603 (2003).

[7] T. Vojta, J. Phys. A 39, R143 (2006).

[8] See e.g. K. McElroy et al., Science 309, 1048 (2005).

[9] M. Milovanovic, S. Sachdev, and R. Bhatt, Phys. Rev. Lett. 63, 82 (1989).

[10] M. Lakner, H. v. Löhneysen, A. Langenfeld, and P. Wölfle, Phys. Rev. B 50, 17064 (1994). 